\title{
Pulmonary Hemorrhage in a Patient with Brain-Lung-Thyroid Syndrome Caused by a p.T86fs Variant in the $N K X 2-1$ Gene
}

\author{
Nina Marić, Olivera Ljuboja \\ Clinic for Children Diseases, University Clinical Centre of the Republic of Srpska, Bosnia and Herzegovina \\ Correspondence: ninamaric.bl@gmail.com; Tel.: + 38751342 216; Fax.: + 38751342316
}

Received: May 6, 2020; Accepted: July 5, 2020

\begin{abstract}
Objective - Brain-lung-thyroid syndrome is a rare disorder caused by mutations in the NKX2-1 gene. The syndrome is characterized by neonatal respiratory distress, choreoathetosis and hypothyroidism, but the clinical spectrum and severity of symptoms vary widely. To our knowledge, diffuse pulmonary hemorrhage has not been previously associated with the NKX2-1 gene. Case report - We report a patient with brain-lung-thyroid syndrome who suffered from pulmonary hemorrhage in the third hour of life, which led to acute respiratory distress and required urgent endotracheal intubation and mechanical ventilation. In addition, our patient has a phenotype that is typical for brain-lung-thyroid syndrome and carries a de novo heterozygous pathogenic a p.T86fs variant in the NKX2-1 gene. Conclusion - Pulmonary hemorrhage in our patient could be a primary manifestation of the brain-lung-thyroid syndrome. This report provides evidence of a wide spectrum of $N K X 2-1$-associated pulmonary phenotypes and underscores the importance of considering NKX2-1-related disorder in the differential diagnosis of pulmonary disease of unknown cause.
\end{abstract}

Key Words: Brain-Lung-Thyroid Syndrome • Pulmonary Hemorrhage • NKX2-1 gene

\section{Introduction}

Brain-lung-thyroid syndrome is a rare disorder characterized by neurological abnormalities, hypothyroidism and neonatal respiratory distress. It is caused by mutations in the NKX2-1 gene, also known as the thyroid transcription factor 1 (TTF1) gene, located on chromosome 14 (locus 14q13). The NKX2-1 gene encodes the thyroid transcription factor 1 (TTF-1), now called NK2 homeobox 1 protein, that has long been recognized for its involvement in lung, thyroid and forebrain embryogenesis. Its importance is demonstrated by the absence of thyroid glands, lungs, pituitary gland and ventral forebrain in TTF-1 knockout mice (1). The first reported human with heterozygous deletion of chromosome 14q13-20, and corresponding ab- sence of the TTF-1 gene, was a full-term infant who developed respiratory failure shortly after delivery, neonatal subclinical hypothyroidism, and exhibited developmental delays, recurrent lower airway infection and atelectasis (2). Since this discovery, numerous other cases with variable combinations of congenital hypothyroidism, neurological features and pulmonary symptoms, related to haploinsufficiency of the NKX2-1 gene, have been reported $(3,4,5,6)$.

The classical triad of symptoms is not always present. In a review of 46 affected individuals it was found that $50 \%$ had full brain-lung-thyroid syndrome (3). The most consistent clinical features of this disorder are neurological abnormalities, such as chorea, ataxia, dystonia and delayed motor development, but also sleep, hunger and thirst disturbances and recurrence of fever due to hypothalamic 
dysfunction. Hypothyroidism, resulting from thyroid dysmorphogenesis, has been reported in $87 \%$ of all cases (3). Pulmonary disease is reported in up to $78 \%$ of patients $(3,7,8)$. It may precede the neurological and thyroid phenotype, but it may also be the only manifestation of the disease (9). It is known that the NKX2-1 gene controls the lung structures' development and regulates the expression of surfactant proteins essential for lung stability and lung host defence $(1,11)$. Patients with NKX2-1 mutations may present in the newborn period with a spectrum of pulmonary manifestations, including severe respiratory distress syndrome with or without pulmonary hypertension, alveolar proteinosis and recurrent respiratory infections $(9,12)$. Moreover, mutations within the NKX2-1 gene may be associated with neuroendocrine cell hyperplasia of infancy, an interstitial lung disease that mostly occurs between ages four months and seven years, and pulmonary fibrosis in older individuals $(9,13$, 14). Affected children also frequently suffer from asthma and recurrent respiratory infections.

Pulmonary hemorrhage in neonates is a rare, lifetreating condition. It is associated with low gestation age, intrauterine growth restriction, intrapartum asphyxia, infections, persistent ductus arteriosus, bleeding disorders, meconium aspiration, resuscitation in the delivery room, surfactant therapy and maternal use of anticonvulsants, vitamin $\mathrm{K}$ antagonists and cocaine (15). It is important to establish an etiological diagnosis of the pulmonary hemorrhage, because its management and prognosis primarily depend on the underlying disorder. We present here a patient with brain-lung-thyroid syndrome caused by a p.T86fs variant in the NKX2-1 gene, who suffered from diffuse pulmonary hemorrhage shortly after birth. To our knowledge, based on a search on the PubMed database using the keywords "pulmonary hemorrhage" and "NKX2-1" or "brain-lung-thyroid syndrome", pulmonary hemorrhage has not been reported in patients with brain-lung-thyroid syndrome.

\section{Case Report}

The five-year-old girl is the second child of healthy, unrelated parents. She was born at term after an uneventful pregnancy by vaginal birth. Apgar scores were 9 and 10 in the first and fifth minutes respectively. Her birth weight was 3080 g. Symptoms of respiratory distress began suddenly in the 3rd hour of life, and required urgent endotracheal intubation and mechanical ventilation. A huge amount of blood was aspirated from the trachea. There were no signs of trauma or infectious diseases, and coagulation disorders were ruled out by the normal results of coagulation function tests, including prothrombin time, activated partial thromboplastin time, international normalized ratio, fibrinogen and platelet count. An initial chest radiograph was consistent with respiratory distress syndrome. It showed decreased transparency of lung tissue, resembling ground-glass, with bilateral pulmonary infiltrates, followed by aggravation and the appearance of mild subsegmental atelectasis in the next few days. Echocardiography revealed mild pulmonary hypertension. The patient was treated with mechanical ventilation for 12 days, and over the next 11 months she received supplemental oxygen support. On the basis of the symptoms and chest imaging studies, neuroendocrine cell hyperplasia was suspected, but lung biopsy was not performed because of its invasiveness. She was treated with corticosteroids for two and a half years with a good response. During her first three years the patient suffered from recurrent respiratory infections, three of which were pneumonia. She received palivizumab as prophylaxis for respiratory syncytial virus infection and pneumococcal vaccine.

She also suffered from mild hypotonia and delayed motor development. Physical therapy started at the age of 6 months. Choreiform movements, generalized dystonia and occasional myoclonic jerks started at the age of 9 months. She was sitting from the 10th month and started to walk at the age of 19 months. Choreiform movements in the extremities and trunk were aggravated during walking and caused her to fall frequently. Episodes of fever, without clinical and laboratory signs of infection, started when she was 12 months old. She had normal speech development and body weight, and no dysmorphic features. Results of brain and 
Father

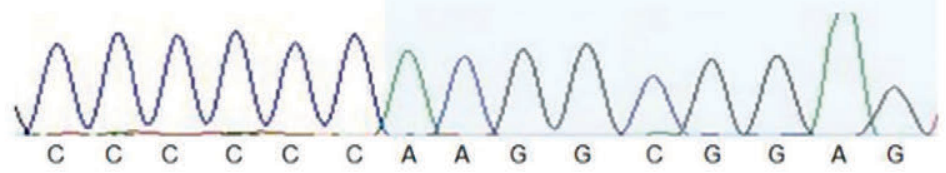

Patient

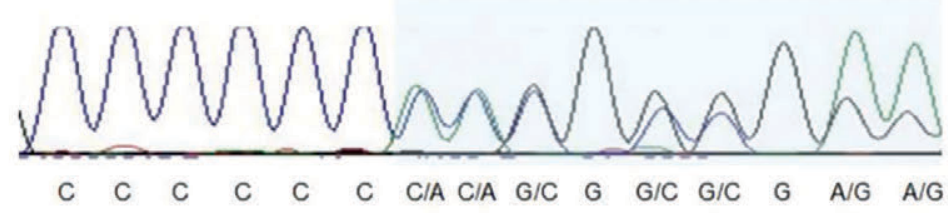

Mother

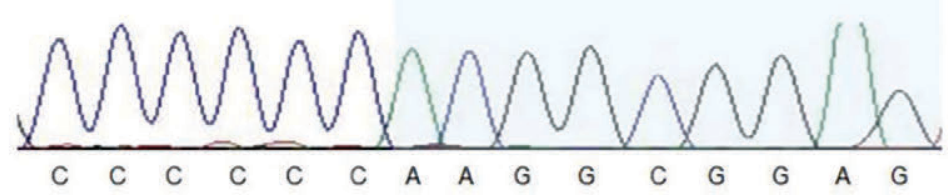

Fig. 1. The image illustrates a portion of the sequencing data obtained for the regions containing the variant for study in blue. A heterozygous small insertion can be observed as the presence of two signals along the sequence. Only the variant c.254dupG:p. T86fs is present in the patient.

spine NMR, EMNG, EEG, echocardiography, serum levels of creatine kinase and tests for metabolic diseases were normal. Neonatal screening did not reveal elevated thyroid-stimulating hormone. There is no family history suggestive of affected relatives.

When the patient was 3 years old, whole-exome sequencing on an Illumina NextSeq platform was performed, after obtaining written informed consent from her parents. The heterozygous frameshift variant c.254dupG, caused by the insertion of one nucleotide at a genomic position [NCBI37] chr14:36,988,308, was detected in the NKX2-1 gene. This variant modifies the messenger RNA reading frame and introduces a termination signal codon at position 405 (p.T86fs). The consequence is the synthesis of an elongated protein of 404 amino acids, instead of 371 if no mutation is present. Sanger sequencing confirmed the presence of the variant p.T86fs in the patient and, since it was not observed in the parental samples, her origin was considered to be de novo (Fig. 1). This variant has been classified as pathogenic and has already been identified in patients with respiratory distress syndrome, chorea and hypothyroidism (12).

After obtaining a genetic diagnosis, we made plans for follow-up and management of the patient by specialists in pediatric pulmonology, neurology and endocrinology. At the age of 3 years, thyroidstimulating hormone was 6.52 (normal limits 0.4 - $4.50 \mathrm{mU} / \mathrm{L}$ ) and free thyroxine 13.55 (normal limits $9-19 \mathrm{pmol} / \mathrm{L}$ ) that was assumed to be subclinical hypothyroidism, and she started to receive levothyroxine replacement therapy. The patient undergoes annual screening for pulmonary and thyroid cancer. In the last two years, the patient has had recurrent bronchospasm attacks. Two attacks, that were induced by a respiratory infection, required hospital treatment. Between attacks, she does not need oxygen support. Pulmonary hemorrhage has not recurred. Chorea seems to be non-progressive and she is now walking with fewer difficulties. She still has episodes of fever not related to infection. 
Her height and body weight are within the normal range, and intelligence is normal.

A clinical geneticist performed counseling for the patient's parents and explained the genetic risks. Since NKX2-1-related disorders are inherited in an autosomal dominant manner and the pathogenic NKX2-1 variant was considered to be de novo in the patient, the empiric risk to siblings is approximately $1 \%$ because of the theoretic possibility of parental germline mosaicism. The risk for the patient's future offspring is $50 \%$. The patient will be advised to undergo to genetic counseling at the age of 16 .

\section{Discussion}

Brain-lung-thyroid syndrome is a rare disorder, characterized by choreoathetosis, hypothyroidism and a spectrum of pulmonary manifestations, caused by mutations in the gene NKX2-1 (3, 4, 5, $6)$. The pulmonary phenotype includes respiratory distress syndrome, alveolar proteinosis, neuroendocrine cell hyperplasia, interstitial lung disease, pulmonary fibrosis, recurrent respiratory infections and asthma $(9,12,13,14)$. Pulmonary hemorrhage has not previously been associated with mutations in the NKX2-1 gene.

We have presented a patient with brain-lungthyroid syndrome, caused by a p.T86fs variant in the NKX2-1 gene, who suffered diffuse pulmonary hemorrhage in the 3rd hour of life, that led to acute respiratory distress and required urgent endotracheal intubation and mechanical ventilation. After that initial event, the patient has not suffered from pulmonary hemorrhage, but she does have other pulmonary manifestations typical for an NKX21 -related disease, as well as exhibiting the NKX21-related neurological phenotype and laboratory signs of hypothyroidism. The etiological diagnosis of the pulmonary hemorrhage was not established and, in our opinion, it could be a rare manifestation of the NKX2-1-related phenotype. There have been some pathological and radiological findings mentioned in the literature that suggest that pulmonary hemorrhage could be a part of an NKX2-1-related disorder. It has been identified in rare cases of this syndrome via a lung biopsy (10). Also, ground-glass and airspace opacities, which are the most common high-resolution computed tomography chest findings in brain-lung-thyroid syndrome, are known results of alveolar hemorrhage (16). Unfortunately, because of a technical problem, we could not show chest $\mathrm{x}$-ray images in our case. A possible pathogenetic mechanism of the pulmonary hemorrhage in our patient is surfactant dysfunction that was suggested to be implicated in the pathogenesis of pulmonary hemorrhage by Amizuka et al. (17). This case, therefore, emphasizes the need to consider an NKX2-1-related disorder in a neonate with pulmonary hemorrhage, after common causes have been ruled out.

Our patient's phenotype includes the full classical triad of symptoms typical for brain-lung-thyroid syndrome. Genetic analysis revealed that the patient has de novo pathogenic heterozygous frameshift variant p.T86fs in the NKX2-1 gene that has been already identified in two patients with respiratory distress syndrome, chorea and hypothyroidism (12). Although pulmonary hemorrhage was not reported in these cases, this variant in the NKX2-1 gene cannot be excluded as its cause, since manifestations vary between individuals with the same NKX2-1 pathogenic variant $(4,7,12)$. Due to the lack of genotype-phenotype correlations, it is not possible to predict the exact course of the disease in our patient or to give her parents proper information regarding prognosis. Our greatest concern is the course of her pulmonary disease, since it is considered to be mainly responsible for mortality in these patients (12). On the other hand, we were able to give the parents the encouraging information that in most studied cases chorea improved up until puberty and stabilized, or was even resolved in adulthood (7). Although rarely, people with brain-lung-thyroid syndrome can have short stature, anomalies of the heart and genitourinary organs and hypodontia. They also have an elevated risk for thyroid and pulmonary carcinoma (7). Accordingly, our patient was referred to the relevant specialists and regularly undergoes the appropriate screening tests. 
It is known that pulmonary disease may precede the neurological and thyroid phenotype, and may also be the only manifestation of the NKX2-1-related disease. Thus, NKX2-1 analysis for patients with isolated pulmonary disease is considered informative and is recommended (9). Genetic diagnosis allowed us to detect other diseases that are part of the NKX2-1 phenotype and that are not yet symptomatic in our patient, and to avoid the invasive diagnostic investigations as lung biopsy. In our case, the results of the neonatal screening test did not reveal congenital hypothyroidism. The repeated test, performed after obtaining the genetic diagnosis, showed slightly elevated thyroid-stimulating hormone with normal free thyroxine value, and the patient started to receive levothyroxine replacement therapy. Hypothyroidism was diagnosed while it still was subclinical, and thus the possible systematic sequelae of hypothyroidism, that would have additionally delayed her development, were prevented. Unfortunately, because of a neurological disease, the patient underwent a large number of investigations that could have been avoided if the genetic test had been done earlier. Therefore, we believe that clinicians should consider testing the NKX2-1 gene when confronted with a child with diffuse lung disease of unknown cause, even though endocrine and neurological system involvement is not present.

\section{Conclusion}

Our case is a very rare example of brain-lung-thyroid syndrome with pulmonary hemorrhage that might be a primary disease manifestation. This report provides evidence of a wide spectrum of NKX2-1 associated pulmonary phenotypes, and underscores the importance of considering an NKX2-1-related disorder in the differential diagnosis of pulmonary disease of unknown cause, especially when endocrine and neurological system involvement is present. Estimating genetic diagnosis allows us to perform proper management and create a followup plan for the patient and give genetic information to the family.
Authors' Contributions: Conception and design: NM and OLJ; Acquisition, analysis and interpretation of data: NM and OLJ; Drafting the article: NM; Revising of the article critically for the intellectual content: OLJ; Approved final version of the manuscript: NM and OLJ

Conflict of Interest: The authors declare that they have no conflict of interest.

Funding: No external funding.

\section{References}

1. Kimura S, Hara Y, Pineau T, Fernandez-Salguero P, Fox $\mathrm{CH}$, Ward JM, et al. The T/ebp null mouse: thyroid-specific enhancer-binding protein is essential for the organogenesis of the thyroid, lung, ventral forebrain, and pituitary. Genes Dev. 1996;10:60-9.

2. Devriendt K, Vanhole C, Matthijs G, de Zegher F. Deletion of thyroid transcription factor-1 gene in an infant with neonatal thyroid dysfunction and respiratory failure. N Engl J Med. 1998;338:1317-18.

3. Carré A, Szinnai G, Castanet M, Sura-Trueba S, Tron E, Broutin-L'Hermite I, et al. Five new TTF1/NKX2.1 mutations in brain-lung-thyroid syndrome: rescue by PAX8 synergism in one case. Hum Mol Genet. 2009;18:226676.

4. Thorwarth A, Schnittert-Hübener S, Schrumpf P, Müller I, Jyrch S, Dame C, et al. Comprehensive genotyping and clinical characterisation reveal 27 novel NKX2-1 mutations and expand the phenotypic spectrum. J Med Genet. 2014;51(6):375-87.

5. De Filippis T, Marelli F, Vigone MC, Di Frenna M, Weber G, Persani L. Novel NKX2-1 frameshift mutations in patients with atypical phenotypes of the brain-lung-thyroid syndrome. Eur Thyroid J 2014;3:227-33.

6. Williamson S, Kirkpatrick M, Greene S, Goudie D. A novel mutation of NKX2-1 affecting 2 generations with hypothyroidism and choreoathetosis: part of the spectrum of brain-thyroid-lung syndrome. J Child Neurol. 2014;29:666-9.

7. Gras D, Jonard L, Roze E, Chantot-Bastaraud S, Koht J, Motte J, et al. Benign hereditary chorea: phenotype, prognosis, therapeutic outcome and long term follow-up in a large series with new mutations in the TITF1/NKX2-1 gene. J Neurol Neurosurg Psychiatry. 2012;83:956-62.

8. Parnes M, Bashir H, Jankovic J. Is Benign Hereditary Chorea Really Benign? Brain-Lung-Thyroid Syndrome Caused by NKX2-1 Mutations. Mov Disord Clin Pract. 2019;6:34-9. 
9. Hamvas A, Deterding RR, Wert SE, White FV, Dishop $\mathrm{MK}$, Alfano DN, et al. Heterogeneous pulmonary phenotypes associated with mutations in the thyroid transcription factor gene NKX2-1. Chest. 2013;144:794-804.

10. Galambos C, Levy H, Cannon CL, Vergas SO, Reid LM, Cleveland R, et al. Pulmonary pathology in thyroid transcription factor-1 deficiency syndrome. Am J Respir Crit Care Med. 2010;182(4):549-54.

11. Kleinlein B, Griese M, Liebisch G, Krude H, Lohse P, Aslanidis C, et al. Fatal neonatal respiratory failure in an infant with congenital hypothyroidism due to haploinsufficiency of the NKX2-1 gene: alteration of pulmonary surfactant homeostasis. Arch Dis Child Fetal Neonatal Ed. 2011;96(6):453-6.

12. Nattes E, Lejeune S, Carsin A, Borie R, Gibertini I, Balinotti J, et al. Heterogeneity of lung disease associated with nk2 homeobox 1 mutations. Respiratory medicine. 2017;129:16-23.

13. Young LR, Deutsch GH, Bokulic RE, Brody AS, Nogee LM. A mutation in TTF1/NKX2.1 is associated with fa- milial neuroendocrine cell hyperplasia of infancy. Chest. 2013;144(4):1199-1206.

14. Guillot L, Carre A, Szinnai G, Castanet M, Tron E, Jaubert $\mathrm{F}$, et al. NKX2-1 mutations leading to surfactant protein promoter dysregulation cause interstitial lung disease in "Brain-Lung-Thyroid Syndrome". Hum Mutat. 2010;31:1146-62.

15. Zahr RA, Ashfaq A, Marron-Corwin M. Neonatal Pulmonary Hemorrhage. NeoReviews. 2012;13(5):302-6.

16. LeMoine BD, Browne LP, Liptzin DR, Deterding RR, Galambos C, Weinman JP. High-resolution computed tomography findings of thyroid transcription factor 1 deficiency (NKX2-1 mutations). Pediatr Radiol. 2019;49(7):869-75.

17. Amizuka T, Shimizu H, Niida Y, Ogawa Y. Surfactant therapy in neonates with respiratory failure due to haemorrhagic pulmonary oedema. Eur J Pediatr. 2003;162(10):697-702. 